\title{
THE USEFULNESS OF OS-MRS IN THE PREDICTION OF HOSPITAL LENGTH OF STAY - A RETROSPECTIVE AUDIT IN A TERTIARY HOSPITAL
}

\section{BACKGROUND AND GOALS}

* Obesity Surgery Mortality Risk Score (OS-MRS) - 90 days mortality in patients submitted to bariatric surgery ${ }^{(1)}$ * 5 factors - 1 point per each:

$\checkmark$ Body Mass Index (BMI) $>50 \mathrm{~kg} / \mathrm{m}^{2}$

$\checkmark$ Arterial Hypertension

$\checkmark$ Male sex

$\checkmark$ Age $>45$ years old

$\checkmark$ Risk factors for pulmonary thromboembolism * The goal of this study was to evaluate the relation between OS-MRS Class and hospital length of stay

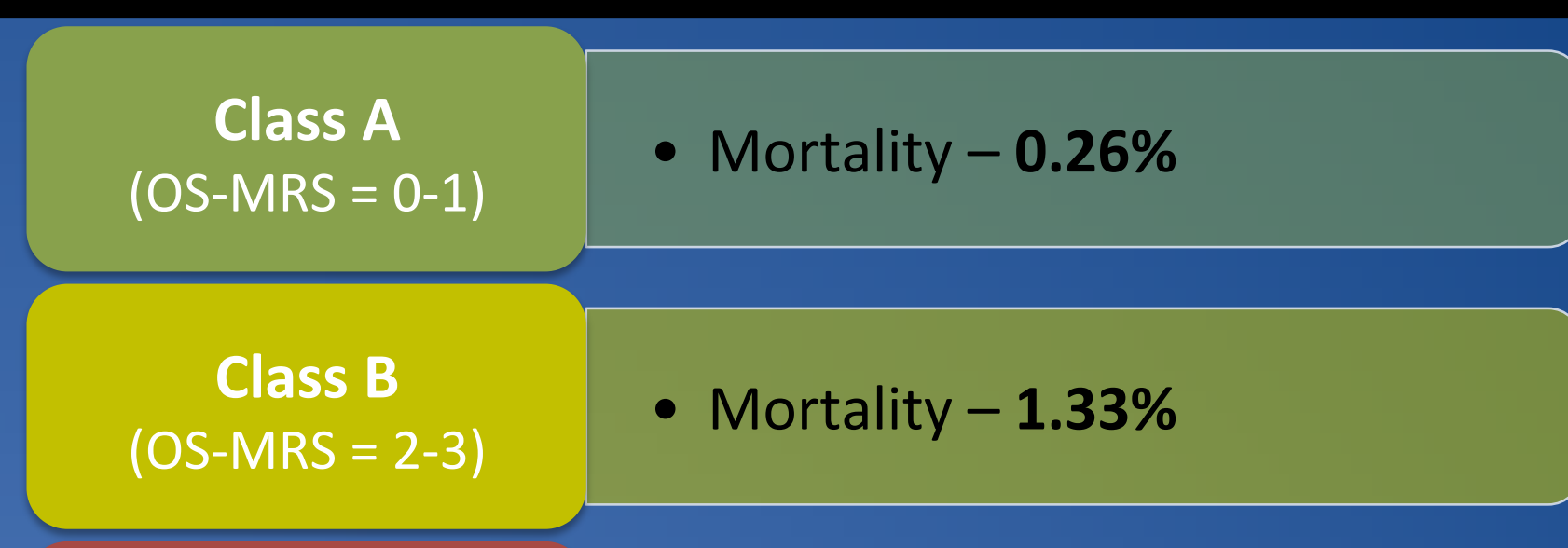

\section{Class C (OS-MRS = 4-5) \\ - Mortality $-4.34 \%$}

Figure 1. OS-MRS Classes and predicted mortality.

\section{MATERIALS AND METHODS}

* Obtained institutional review board approval

* Retrospective audit to patients submitted to elective gastric bypass surgery from January 2010 to May 2015

* Age, gender, American Society of Anaesthesiology (ASA) Physical Status Classification, BMI, OS-MRS, surgery time and hospital length of stay were collected (Table 1)

* Patients classified as OS-MRS, Class A, Class B or Class C

* Kruskal-Wallis with Dunn post-hoc test $(p<0.05)$ was used to compare groups based on OS-MRS (Classes A, B and C)

* Results presented as percentage or mean \pm standard deviation

\section{RESULTS}

\begin{tabular}{|c|c|c|c|c|}
\hline OS-MRS & Class A $(n=168)$ & Class B $(n=184)$ & Class C $(n=4 I)$ & Total $(n=393)$ \\
\hline Age (years old) & $36.8( \pm 7.8)$ & $48.8( \pm 9.6)$ & $51.1( \pm 7.24)$ & $43.9( \pm 10.6)$ \\
\hline Gender [F/M] (\%) & $97.6 / 2.4$ & $80.4 / 19.6$ & $26.8 / 73.2$ & $82.2 / 17.8$ \\
\hline ASA status [2/3] (\%) & $24.2 / 75.8$ & $20.8 / 79.2$ & $8.0 / 92.0$ & $20.9 / 79.1$ \\
\hline $\mathrm{BMI}\left(\mathrm{kg} / \mathrm{m}^{2}\right)$ & $43.4( \pm 6.6)$ & $44.4( \pm 6.0)$ & $46.6( \pm 7.7)$ & $44.2( \pm 6.4)$ \\
\hline Surgery time (min.) & $126.6( \pm 35.7)$ & I $33.6( \pm 39.8)$ & $15 \mid .5( \pm 52.1)$ & $132.5( \pm 40.2)$ \\
\hline Hospital length of stay (days) & $3.2( \pm 1.0)$ & $3.5( \pm 1.2)$ & $3.8( \pm 1.3)$ & $3.4( \pm I .1)$ \\
\hline
\end{tabular}

Table 1. Characterization of the sample.

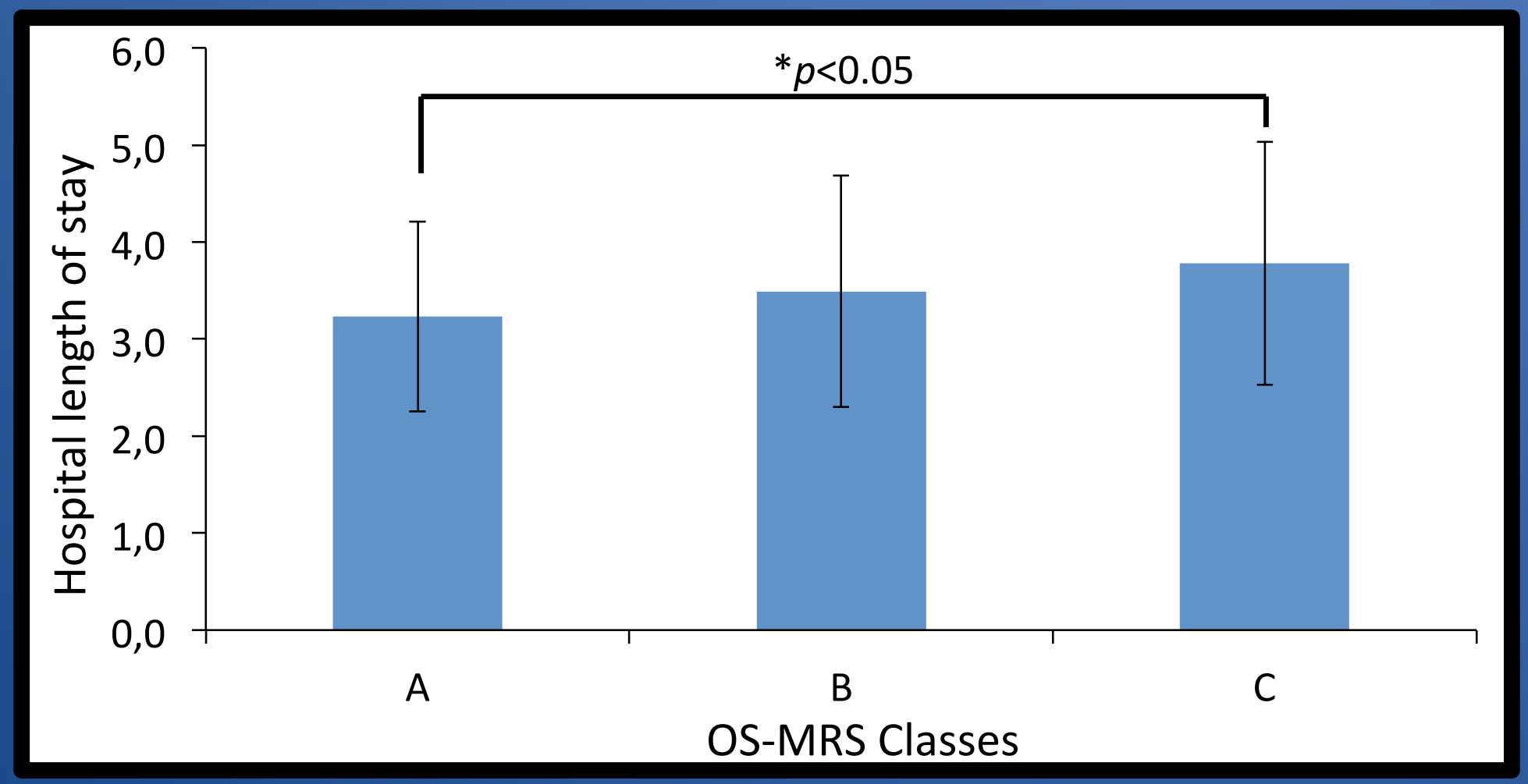

Figure 2.

Hospital length of stay per OS-MRS Classes.

\section{CONCLUSIONS}

* It seems to be a positive relation between OS-MRS classification and hospital length of stay.

* Significant difference between OS-MRS Class A and Class C.

* No significant difference between OS-MRS Class A and Class B and between OS-MRS Class B and Class C.

* OS-MRS classification may be used with caution to predict hospital length of stay. 